\title{
Giardíase e criptosporidiose em crianças institucionalizadas em creches no Estado de São Paulo
}

\author{
Giardiasis and cryptosporidiosis in children institutionalized at \\ daycare centers in the State of São Paulo
}

\author{
Luciene Maura Mascarini ${ }^{1}$ e Maria Rita Donalísio ${ }^{2}$
}

\begin{abstract}
RESUMO
Realizou-se dois estudos transversais em 2002 ( N=379) e 2003 ( N=397) onde estimou-se a prevalência de enteroparasitas em crianças de cinco creches municipais de Botucatu, SP. Coletou-se variáveis socioeconômicas, sanitárias e educacionais e realizou-se exames coproparasitológicos. Giardia duodenalis apresentou prevalência de 23,7\% (2002) e 21,4\% (2003) seguido por Cryptosporidium sp com 15,5\% (2002) e 3,7\% (2003).
\end{abstract}

Palavras-chaves: Giardíase. Cryptosporidiose. Crianças.

\begin{abstract}
Two cross-sectional studies were conducted, in $2002(N=379)$ and $2003(N=397)$, to estimate the prevalence of intestinal parasites among children at five municipal daycare centers in Botucatu, State of São Paulo. Socioeconomic, sanitary and educational data were collected and parasitological tests were performed on feces. Giardia duodenalis presented prevalence of $23.7 \%$ (2002) and 21.4\% (2003) followed by Cryptosporidium sp with 15.5\% (2002) and 3.7\% (2003).
\end{abstract}

Key-words: Giardiasis. Cryptosporidiosis. Children.

Nas últimas décadas, têm-se registrado profundas mudanças na força de trabalho da população em diversas cidades, tendo como resultado um grande número de crianças sendo cuidadas fora do ambiente familiar, institucionalizadas em creches $^{3}$. Além da natural vulnerabilidade desse segmento etário, a criança usuária de creche tem maior probabilidade de adquirir e desenvolver infecções, sobretudo as de repetição, como as respiratórias, gastrointenstinais e cutâneas ${ }^{21}$. Diarréias e gastroenterites são consideradas um importante problema de saúde pública em crianças atendidas em creches $^{520}$.

A giardíase, causada pelo protozoário Giardia duodenalis (sinonímia Giardia intestinalis/Giardia lamblia) e a criptosporidiose causada por Cryptosporidium sp, são enteroparasitoses, muito comuns, manifestadas clinicamente com diarréias e gastroenterites em creches ${ }^{510}$. Estes agentes são transmitidos pelo contato oral/fecal ou por ingestão de alimentos e água contaminados pelas formas infectantes (cistos e oocistos $)^{117}$. Este estudo teve como objetivo contribuir para o conhecimento dos aspectos epidemiológicos das protozooses: giardíase e criptosporidiose em crianças menores de sete anos institucionalizadas em creches no município de Botucatu/SP, Brasil.

0 estudo foi realizado no município de Botucatu/SP, localizado na região centro-oeste do Estado de São Paulo, possuindo, pelo censo de 2000, 108.112 habitantes $^{12}$. 0 município tem 17 creches: três de região central, $12 \mathrm{em}$ bairros periféricos e duas na zona rural. A distribuição das crianças dentro das creches é feita por faixa etária: berçário (0- 24 meses), mini-grupo (25- 39 meses), maternal (40-52 meses), jardim (53-71 meses) e pré-primário (72-83 meses).

Foram realizados dois estudos transversais nos anos de $2002(\mathrm{~N}=379)$ e $2003(\mathrm{~N}=397)$ sendo a creche, a unidade de pesquisa. Foram sorteadas cinco instituições do município (amostragem aleatória estratificada garantindo a representação proporcional das regiões): uma na zona rural (creche de Vitoriana) e quatro na zona urbana (na região

\footnotetext{
1. Departamento de Parasitologia do Instituto de Biociências da Universidade Estadual Paulista (UNESP), Botucatu, SP. 2. Departamento de Medicina Preventiva e Social da Faculdade de Ciências Médicas da Universidade Estadual de Campinas, Campinas, SP.

Endereço para Correpondência: Prof ${ }^{a}$ Luciene Maura Mascarini. Dept ${ }^{0}$ de Parasitologia/ IB/UNESP. Rubião Jr s/n, Caixa Postal 510, Câmpus de Botucatu, 18618-100 Botucatu, SP. Fax: 5514 3815-3744

e-mail: luciene@ibb.unesp.br

Recebido para publicação em 17/10/2005

Aceito em 4/12/2006
} 
central - creche 'AAMI' e três na área periférica (Horestes Spadotto, Cohab e Jardim Flamboyant) da cidade. 0 tamanho da amostra de crianças foi calculado levando-se em consideração a prevalência de enteroparasitoses observada em estudo anterior (prevalência de 64\%) ${ }^{2}$. Estabeleceu-se a prevalência em $50 \%$, com variação de $\pm 5 \%$, com erro amostral de 5\%, estipulando-se o nível de $95 \%$ de significância.

Foram coletadas 3 amostras de fezes de cada criança e confeccionado pull fecal, onde foram realizados exames coproparasitológicos pelos métodos de Faust, Lutz e ZiehlNeelsen modificado ${ }^{11}$. Aplicou-se questionários nos pais ou responsáveis pelas crianças, onde foram coletadas variáveis sócio-econômicas, sanitárias e educacionais.

Foi realizada análise univariada $\left(\chi^{2}\right)$ no programa EPI INFO-6 ${ }^{6}$, entre a variável dependente (presença de enteroparasitas) e as covariáveis: condições sócioeconômicas, sanitárias, educacionais e presença de animais domésticos. A estratificação das variáveis foram: na faixa etária seguiu-se a distribuição das crianças nas salas, a renda familiar foi agrupada em: <2 salários; 2 a 4 salários e $>4$ salários, a moradia em famílias que residiam em zona rural e zona urbana e a escolaridade da mãe em nível $1=$ sem educação formal e $1^{\circ}$ grau incompleto; nível $2=1^{\circ}$ grau completo e $2^{\circ}$ grau incompleto e nível $3=2^{\circ}$ grau completo e superior incompleto e completo.

0 consentimento para a realização dos exames foi obtido formalmente dos pais ou de seus responsáveis e o estudo foi aprovado pelo Comitê de Ética em Pesquisa com seres humanos da Faculdade de Medicina de Botucatu, Universidade Estadual Paulista - São Paulo, Brasil.

Foram analisadas 379 crianças, em 2002, e 397, em 2003, com coeficientes de prevalência discriminados na Tabela 1 . As creches foram estatisticamente diferentes, quanto à presença de enteroparasitas $\left(\chi^{2}=40,74 \mathrm{p}<0,001\right.$, em 2002 e $\chi^{2}=39,61$; $\mathrm{p}<0,001$ em 2003), destacando-se a creche de Vitoriana que apresentou em ambos os anos prevalência maior (76,7 e 51,3\%). 0 protoparasita mais prevalente, em ambos os anos (2002 e 2003), foi $G$. duodenalis com prevalência de 23,7\% (2002) e 21,4\% (2003), seguido por Cryptosporidium sp (15,5\% em 2002 e 3,7\% em 2003) e Blastocystis hominis (13,1\% em 2002 e 5,5\% em 2003) (Tabela 2).

Tabela 1 - Prevalência de enteroparasitas nas 5 creches municipais de Botucatu/SP, nos anos de 2002 e 2003.

\begin{tabular}{|c|c|c|c|c|c|c|}
\hline \multirow[b]{2}{*}{ Creches } & \multicolumn{2}{|c|}{ Total $\left(n^{0}\right)$} & \multicolumn{2}{|c|}{ Positivos $\left(\mathrm{n}^{0}\right)$} & \multicolumn{2}{|c|}{$\begin{array}{c}\text { Coeficiente } \\
\text { prevalência/100 }\end{array}$} \\
\hline & 2002 & 2003 & $2002^{a}$ & $2003^{b}$ & 2002 & 2003 \\
\hline Horestes Spadoto (VF) & 43 & 73 & 27 & 26 & 62,8 & 35,6 \\
\hline Vitoriana (VI) & 86 & 113 & 66 & 58 & 76,7 & 51,3 \\
\hline AAMI (VL) & 90 & 65 & 31 & 04 & 34,4 & 6,1 \\
\hline Cohab (CO) & 62 & 48 & 24 & 19 & 38,7 & 39,6 \\
\hline J. Flamboyant (JF) & 98 & 98 & 43 & 28 & 43,9 & 28,6 \\
\hline Total & 379 & 397 & 191 & 135 & 50,4 & 34,0 \\
\hline
\end{tabular}

Tabela 2 - Prevalência de cada enteroparasita encontrado nas 5 creches municipais de Botucatu/SP, nos anos de 2002 e 2003.

\begin{tabular}{|c|c|c|c|c|}
\hline \multirow{3}{*}{ Parasitas } & \multicolumn{2}{|c|}{$\mathrm{N}^{\circ}$ total } & \multirow{2}{*}{\multicolumn{2}{|c|}{$\begin{array}{c}\text { coeficiente } \\
\text { prevalência / } 100\end{array}$}} \\
\hline & \multirow{2}{*}{$\begin{array}{l}\mathrm{N}=379 \\
2002\end{array}$} & \multirow{2}{*}{$\frac{N=397}{2003}$} & & \\
\hline & & & 2002 & 2003 \\
\hline \multicolumn{5}{|l|}{ Protozoários } \\
\hline Giardia duodenalis & 90 & 85 & 23,7 & 21,4 \\
\hline Cryptosporidium sp & 59 & 15 & 15,6 & 3,8 \\
\hline Blastocystis hominis ${ }^{1}$ & 50 & 22 & 13,2 & 5,5 \\
\hline Entamoeba coli $^{2}$ & 25 & 18 & 6,6 & 4,5 \\
\hline Endolimax nana ${ }^{2}$ & 7 & 8 & 1,8 & 2,0 \\
\hline Isospora sp & 1 & 0 & 0,3 & 0 \\
\hline Helmintos & 63 & 36 & 16,6 & 9,1 \\
\hline Negativos & 188 & 262 & & \\
\hline
\end{tabular}

${ }^{1}$ espécie categoria controversa; ${ }^{2}$ espécies comensais

Em 2002, a distribuição de Giardia duodenalis e Cryptosporidium sp foi heterogênea nas diferentes faixas etárias $\left(\chi^{2}=14,6(\mathrm{p}=0,005)\right.$ e $\chi^{2}=10,4(\mathrm{p}=0,033)$ sendo que a faixa etária maternal apresentou maior prevalência de parasitismo para ambos os protoparasitas. 0 protozoário G. duodenalis apresentou diferença quanto à sua distribuição dentro das faixas de renda familiar $\left(\chi^{2}=7,5 ; p=0,023\right)$, com $57,9 \%$ de crianças parasitadas na faixa salarial ' 2 a 4 salários'. Quanto à moradia, constatou-se que ambos os protoparasitas têm prevalência maior na zona urbana, quando comparados à zona rural $\left(\chi^{2}=17,8 \mathrm{p}<0,001\right.$ para $G$. duodenalis e $\chi^{2}=18,09 \mathrm{p}<0,001$ para Cryptosporidium sp). A escolaridade da mãe foi uma variável que se apresentou associada à presença de $G$. duodenalis $\left(\chi^{2}=9,5 \mathrm{p}=0,008\right)$ com prevalência maior (59\%) deste protozoário entre as crianças cujas mães possuíam nível de escolaridade 1.

Em 2003, também constatou-se distribuição heterogênea de $G$. duodenalis nas diferentes faixas etárias, com $\div^{2}=10,3$ $(\mathrm{p}=0,03)$. A faixa etária maternal foi a que apresentou maior prevalência com coeficiente de prevalência de 34,1\%, seguido pela faixa etária berçário, com $29,4 \%$ de positividade. 0 protozoário $G$. duodenalis apresentou diferença quanto à sua distribuição dentro das faixas de renda familiar $\left(\chi^{2=16,9 /}\right.$ $\mathrm{p}<0,001)$, com $49,4 \%$ de crianças parasitadas na faixa salarial '2 a 4 salários'.

Em creches, a prevalência das enteroparasitoses em várias cidades do Brasil varia grandemente, atingindo valores elevados em São Paulo: Botucatu (64\%) e Mirassol $(63,9 \%)^{2}{ }^{14}$, em Sergipe (Aracaju com 56,6\%) ${ }^{4}$, decrescendo no Paraná (Rolandia com 15,2\%) ${ }^{9}$. A prevalência de giardíase no Brasil varia de 4 a $30 \%^{4}{ }^{10}$, sugerindo em creches, um padrão de infecção similar ao das infecções bacterianas entéricas, introduzidas por uma única criança, transmitindo-se rapidamente e permanecendo no ambiente servindo como fonte para futuras infecções ${ }^{21}$. A presença de $G$. duodenalis associada à idade observada neste trabalho pode estar relacionada ao aumento na transmissão fecal-oral de patógenos bem como à presença de hábitos higiênicos precários observados nesta faixa etária ${ }^{1}$. A melhoria no nível de escolaridade da mãe, independente do aumento da renda familiar justificaria o declínio observado para a giardíase na 
cidade de São Paulo, constatado nos anos de 1984-19967 , sendo que o nível de escolaridade da mãe influenciaria na introdução e manutenção das práticas higiênicas dos seus filhos ${ }^{19}$. Cryptosporidium sp foi verificado em crianças brasileiras institucionalizadas ou não, com prevalências que variavam de $1,1 \%$ a $20 \%$ e cujas faixas etárias observada por diversos autores ${ }^{161318}$ corroboraram nosso estudo. Este estudo não encontrou associação entre presença de animais domésticos no domicílio e crianças positivas para Cryptosporidium sp e/ ou $G$. duodenalis e apesar de muitos relatos, o potencial zoonótico destes protozoários é ainda hoje discutível ${ }^{15}$. A sazonalidade marcante de Cryptosporidium sp em meses ou estações chuvosas é apontada por autores como fator de risco extrafamiliar na aquisição deste enteroparasita ${ }^{16}{ }^{19}$ não sendo, contudo observada neste estudo.

\section{REFERENCES}

1. Adam RD. Biology of Giardia lamblia. Clinical Microbiology Review 14: 447-475, 2001.

2. Andrade JB, Mascarini LM. Prevalência de enteroparasitas e padrão nutricional em crianças de creches municipais de Botucatu/SP. Jornal Brasileiro de Patologia 37(supl 4): 509, 2001

3. Barros AJ, Ross DA, Fonseca WV, Williams LA, Moreira-Filho DC. Preventing acute respiratory infections and diarrhoea in child care centers. Acta Pediatric 88:1113-8, 1999

4. Cardoso GS, Santana ADC, Aguiar CP. Prevalência e aspectos epidemiológicos da giardíase em creches do município de Aracaju, SE, Brasil. Revista da Sociedade Brasileira de Medicina Tropical 8: 25-31, 1995.

5. Cordell RL, Adiss DG. Cryptosporidiosis in child care settings. Paediatric Infectious Disease Journal 13:310-317, 1994.

6. Dean AG, Dean JÁ, Coulombier D, Brendel KA, Smith DC, Burton AH. Epiinfo Version 6: a Word-Processing, Database and Statistics Program for Public Health on IBM - Compatible Microcomputers. Atlanta: C D C 1995.

7. Ferreira MU, Ferreira CS, Monteiro CA. Tendência secular das parasitoses intestinais na infância na cidade de São Paulo (1984-1996). Revista de Saude Publica 34(supl 6): 73-83, 2000.
8. Franco RMB, Cordeiro NS. Giardiose e criptosporidiose em creches no município de Campinas, SP. Revista da Sociedade Brasileira de Medicina Tropical 29: 585-591, 1996.

9. Giraldi N, Vidotto 0, Navarro IT, Garcia JL. Enteroparasites prevalence among day care and elementary school children of municipal schools, Rolandia, PR, Brazil. Revista da Sociedade Brasileira de Medicina Tropical 34: 385-387, 2001.

10. Guimarães S, Sogayar MIL. Ocurrence of Giardia lamblia in children of municipal day-care centers from Botucatu, São Paulo state, Brazil. Revista do Instituto de Medicina Tropical de Sao Paulo 37: 501-506, 1995.

11. Henriksen A, Pohlenz JFL. Staining of Cryptosporidium by a modified ZiehlNeelsen Technique. Acta Veterinary Scandnavya 22:594-596, 1981.

12. Instituto Brasileiro de Geografia e Estatística. Dados em pesquisa populacional brasileira. <http:WWW/home/estatística/população/ estimativa 2004>. Acesso em 10/0utubro/2004.

13. Loureiro EC, Linhares AC, Mata L. Criptosporidiose em crianças de 1 a 2 anos de idade, com diarréia aguda em Belém, Pará, Brasil. Memórias do Instituto Oswaldo Cruz 84: 117-122, 1989.

14. Machado RC, Marcari EL, Cristante SFV, Carareto CMA. Giardíase e helmintíases em crianças de creches e escolas de $1^{\circ}$ e $2^{\circ}$ graus (públicas e privadas) da cidade de Mirassol (SP, Brasil). Revista da Sociedade Brasileira de Medicina Tropical 32: 1-12, 1999.

15. Monis PT, Thompson RCA. Cryptosporidium and Giardia-zoonoses: fact or fiction? Infectious Genetic Evoluation 3:233-244, 2003.

16. Newman RD, Sears CL, Moore SR, Nataro JP, Wuhib T, Agnwe DA, Guerrant RL, Lima AML. Longitudinal study of Cryptosporidium infection in Children in Northeastern Brazil. The Journal Infectious Disease 180:167-175, 1999.

17. O'donogue PJ. Cryptosporidium and cryptosporidiosis in man and animals. International Journal of Parasitology 25:139-195, 1995.

18. Oshiro ET, Dorval MEC, Nunes VLB, Silva AA, Said LAM. Prevalência do Cryptosporidium parvum em crianças abaixo de 5 anos, residentes na zona urbana de Campo Grande, MS, Brasil. Revista da Sociedade Brasileira de Medicina Tropical 33: 277-280, 2000.

19. Pereira MGC, Atnill ER, Barbosa AP, Silva AS, Garcia-Zapata MTA. Intrafamilial and extra-familial risk factors associated with Cryptosporidium parvum infection among children hospitalized for diarrhea in Goiânia, Goiás, Brazil. American Journal Tropical Medicine and Hygiene 66: 787-793, 2002.

20. Silva LR. Diarréia aguda em crianças até cinco anos. Um estudo epidemiológico, etiolólogico e clínico em Salvador, Bahia. Revista da Sociedade Brasileira de Medicina Tropical 22: 107-108, 1989.

21. Thompson RCA. Giardiasis as a re-emerging infectious disease and its zoonotic potential. International Journal of Parasitology 30: 1259-1267, 2000. 